\title{
Effects of Cement Bases on the Stresses in Amalgam Restorations
}

\author{
J. W. FARAH, J. A. A. HOOD,* and R. G. CRAIG \\ School of Dentistry, University of Michigan, Ann Arbor, Michigan, 48104, USA
}

A computerized dental model was used to study the stress induced in a Class 1 amalgam restoration when supported by bases of vary. ing materials and thicknesses. Under the same load, the maximum tensile stresses and deflections in the amalgam restoration in. creased at least threefold with a zinc oxideeugenol base as compared with a $\mathrm{ZnPO}_{4}$ cement base.

It is a recommended procedure in restorative dentistry to place an intermediary cement base under deep preparations for amalgam restorations. These bases are of low thermal conductivity and serve to protect the vital tissues from thermal shock. A further requirement is that the base have sufficient strength to withstand both the forces of condensation and mastication transmitted to it through the restoration.

The ability of cement bases to withstand condensation forces has been investigated by a number of authors ${ }^{1-3}$ and it was determined that a minimum compressive strength of 170 psi was necessary to prevent displacement of the base during condensation. ${ }^{2}$ There is a general belief that zinc oxideeugenol (ZOE) cements do not have adequate strength to support amalgam restorations, but as stated by El-Bahloul ${ }^{4}$ the strength required in a cement base under a functional amalgam restoration has not been determined.

A variety of mechanical models has been used to investigate the ability of bases to

This investigation was supported by USPHS Research Grant DE-01817 and by International Postdoctoral Research Fellowship TW-01854 from the $\mathrm{Na}$ tional Institute of Dental Research, National Institutes of Health, Bethesda, Md.

Received for publication March 8, 1974.

Accepted for publication June 7, 1974.

- Present address: University of Otago Dental School, Dunedin, New Zealand.

10 support amalgam restorations. Hoppenstand and McConnell ${ }^{5}$ placed amalgam restorations over cement bases in Class 1 cavities prepared in Ivorine a teeth and subjected them to static loading. Rowe ${ }^{6}$ used teeth with a Class 2 preparation, cast from Neyb technical metal, which he filled with amalgam and a variety of supporting bases and subjected them to static loading. Impact loading on amalgam supported by a variety of bases was investigated by Luke $^{7}$ using Class 1 cavity preparations in Plexiglas rods. Stern $^{8}$ used acrylic teeth with Class 2 cavity preparations to investigate the effects of impact loading on the restored tooth.

The most recent study, by Vieira and Mondelli, ${ }^{9}$ used a Class 2 MOD cavity preparation in cast chrome-cobalt teeth that were restored with amalgam and cement bases and subjected to static loading. These systems used the fracture strength of the amalgam under load as an index of the support provided by the bases. Variations in the model systems particularly pertaining to materials used in place of the natural tooth and the difficulty in reproducing the loading on the surface of the amalgam restoration, have precluded comparisons between the various studies and the in vivo situation.

A recently introduced model, ${ }^{10}$ a computer simulation of a natural sized, three-dimensional tooth crown of slightly idealized morphology containing a Class 1 cavity preparation, has been used to investigate stresses and deflections in cavity floors under various conditions. This model is capable of accounting for the mechanical properties of a natural tooth and is independent of mechanically induced loading variables encountered in previous systems. In this investigation the

a Columbia Dentoform Corp., New York, NY.

b The J. M. Ney Co., Hartford, Conn. 
model has been modified to study stresses induced in a Class 1 amalgam restoration supported by bases of varying materials and thicknesses.

\section{Materials and Methods}

A first molar was idealized by an axisymmetric model and was analyzed by the finite element method. ${ }^{11}$ The morphology, as well as the composition of the model, was constructed to reflect the actual properties of dental tissues (Fig 1). A cavity preparation $4.5 \mathrm{~mm}$ in diameter and $4.0 \mathrm{~mm}$ deep, the depth being measured from the occlusal fossa of the model to the base of the cavity floor, was incorporated into the model. Various combinations of cement bases, cementbase thicknesses, and an amalgam restoration of constant dimensions were simulated in the cavity preparation and subjected to $45 \mathrm{~kg}$ instantaneous loading to minimize the viscoelastic behavior of dentin. The load was axisymmetrically applied and annularly concentrated, as shown in planar form in Figure 1.

The following situations were simulated and examined: (1) amalgan restoration to $C-D$ (Fig 1), supported by dentin; (2) amalgam restoration to $C-D$, supported by $2 \mathrm{~mm} \mathrm{ZnPO}_{4}$ cement base; (3) amalgam restoration to $C-D$, supported by $2 \mathrm{~mm} \mathrm{ZOE}$ cement base; (4) amalgam restoration to $C-D$, supported by $1 \mathrm{~mm} \mathrm{ZnPO}_{4}$ over $1 \mathrm{~mm}$ ZOE cement bașe; (5) amalgam restoration

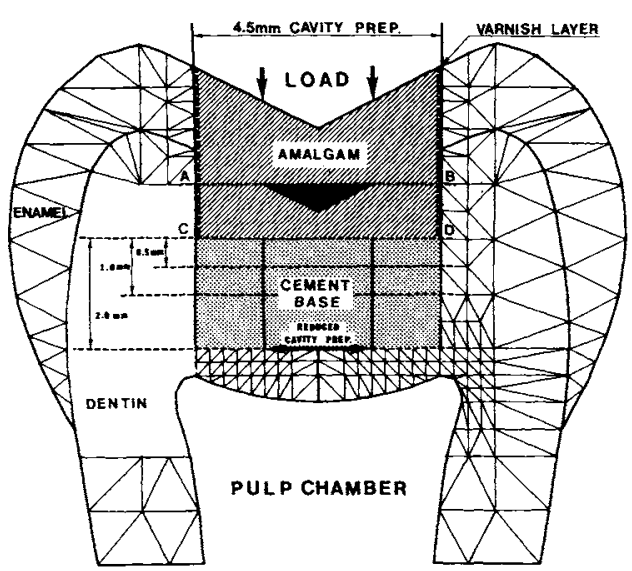

FIg 1.-Axisymmetric model of molar with amalgam restoration supported by various cement bases.

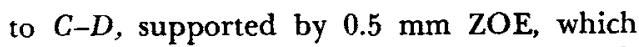
in turn was supported by dentin; (6) amalgam restoration to $C-D$, supported by a dentin floor that contained a reduced cavity in its central part (an excavation $2 \mathrm{~mm}$ in diameter filled with a $2 \mathrm{~mm}$ thickness of ZOE cement base); (7) a preparation similar to (6) except that $\mathrm{ZOE}$ was replaced by $\mathrm{ZnPO}_{4}$ cement base; (8) amalgam restoration down to line $A-B$, supported by some enamel (crowded cross hatch lines in Fig 1) and the rest of the cavity being dentin; and (9) as in (8) with the enamel completely deleted.

In all of these circumstances a layer of varnish was simulated at the cavity wall as shown in Figure 1 , and in one instance the varnish was deleted and its effects on the stresses were analyzed.

The moduli of elasticity and Poisson's ratios used for the calculation of the stresses were: enamel, $82,500 \mathrm{MN} / \mathrm{m}^{2}$ and 0.33 ; dentin, $18,600 \mathrm{MN} / \mathrm{m}^{2}$ and 0.31 ; amalgam, $69,000 \mathrm{MN} / \mathrm{m}^{2}$ and 0.35 ; ZOE cement base, $1,700 \mathrm{MN} / \mathrm{m}^{2}$ and $0.35 ; \mathrm{ZnPO}_{4}$ cement base, $22,400 \mathrm{MN} / \mathrm{m}^{2}$ and $0.35 .12,13$ The maximum shearing, compressive, and tensile stresses, as well as the deflections, just above the amalgam-base interface (denoted by line $C-D)$ were calculated as explained earlier. ${ }^{10}$

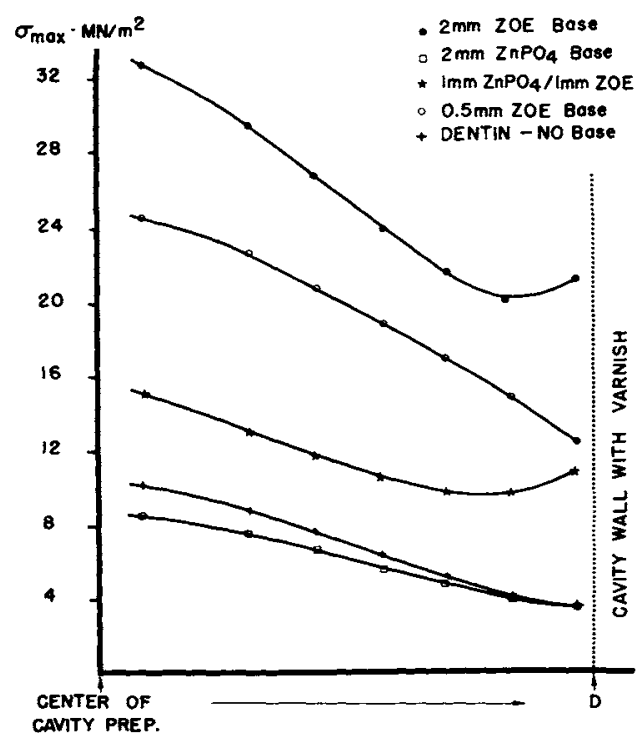

Fig 2.-Maximum tensile stresses in amalgam along line $C-D$, supported by various cement bases. 


\section{Results}

The cavity preparation, as well as the various cement base thicknesses, are presented in Figure 1. In Figure 2 the maximum tensile stresses in the amalgam, adjacent to the amalgam-cement base interface, were plotted as a function of the radius. Figure 2 illustrates the difference in magnitude of the tensile stresses induced in a horizontal plane in the amalgam supported by a cement base of constant thickness $(2 \mathrm{~mm})$ with different mechanical properties. The plots of maximum tensile stress started at the center of the cavity and extended toward the cavity wall. Because of the symmetry of the model, and the symmetry in loading, the stress distribution was radially symmetric around an axis passing through the center of the cavity. In Figure 3 the stress gradient across the interface between the amalgam and either the $\mathrm{ZnPO}_{4}$ or the $\mathrm{ZOE}$ cement base is shown. The stress gradient increased from threefold to fourfold when the ZOE cement base replaced the $\mathrm{ZnPO}_{4}$ base. In Figure 4 , the maximum tensile stress at the amalgam-base interface was plotted for the instance in which the dentin floor contained a reduced cavity in its central part (an excavation $2 \mathrm{~mm}$ in diameter; Fig 1). The maximum tensile stress followed the same trend as in Figure 1. In this case the slope of the stress curve was accentuated; this largely was due to the rapid change in material properties. In Figure 5 the maximum deflection along line $C-D$ was plotted

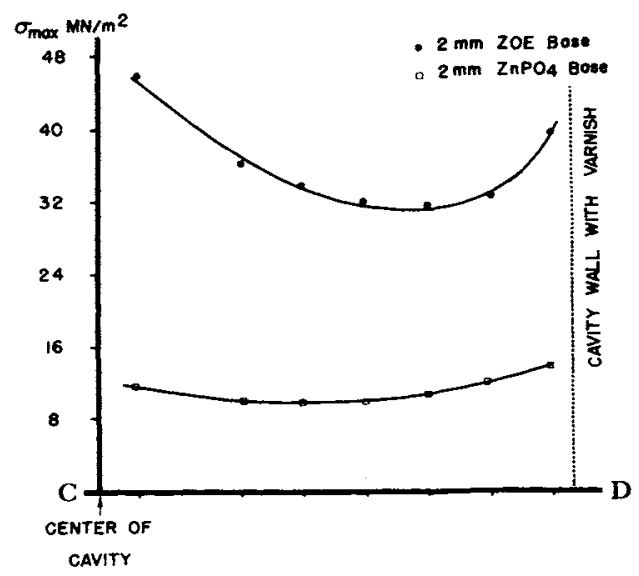

FIf 3.-Stress gradient across line $C-D$ at amalgam-base interface. to demonstrate the effect of the base on the deflection in the amalgam. The deflection increased by more than three and a half times when the $\mathrm{ZnPO}_{4}$ cement base was replaced by the ZOE base. Because of the size and location of the finite element grid in the enamel (case 8) no attempt was made to plot a curve for the distribution of the stresses in that area. However, near the center of the cavity preparation just above the enamel-amalgam interface (line $\boldsymbol{A}-\boldsymbol{B}$ ) a drop of almost $100 \%$ in the tensile stress occurred when the enamel was supporting part of the amalgam.

\section{Discussion}

An analysis of the restored tooth by Tylman 14 indicates that a Class 1 restoration under load behaves as a beam. The yielding floor of dentin or cement under the restoration serves to introduce bending stresses in the restoration and place its lower border in tension. For materials of low tensile strength this lack of support might be sufficient to induce fracture. Experimental results from the model system used by $\mathrm{Luke}^{7}$ indicate that fracture is induced at the amalgam-base interface and propagates to the occlusal surface. This study uses the induced stresses in the amalgam adjacent to the amalgam-base interface as an index of the supporting ability of the base material and hence its ability to prevent tensile fracture of the restoration.

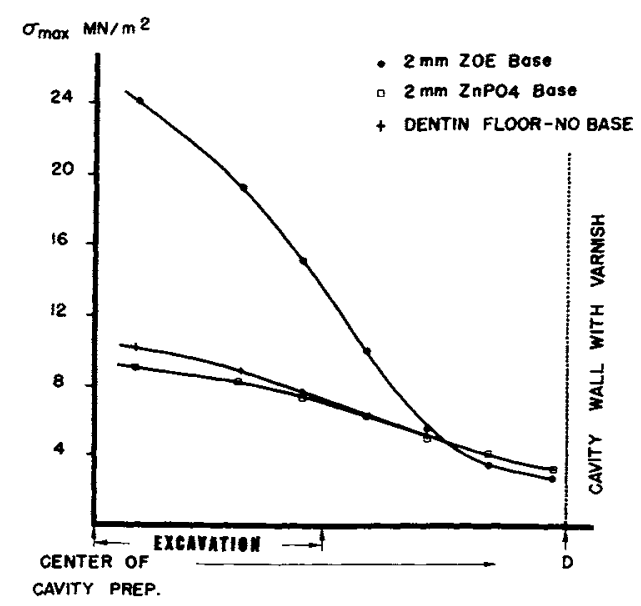

FIG 4.-Maximum tensile stresses in amalgam along line $C-D$, supported by various bases in reduced cavity. 
The model chosen here indicated that the greatest support was provided by a $\mathrm{ZnPO}_{4}$ base, followed by ZOE, and is in agreement with the findings of Hoppenstand and MCConnell, 5 Rowe, 6 and Luke. 7

The present study shows that the tensile stress induced in the amalgam restoration was from four to five times higher when the amalgam was supported by $2 \mathrm{~mm}$ ZOE cement base, as compared with an equal thickness of $\mathrm{ZnPO}_{4}$ cement base (Fig 2). When the stresses induced in the amalgam by a $\mathrm{ZnPO}_{4}$ base of $2 \mathrm{~mm}$ are considered in relation to those induced by a dentin floor alone it can be seen that replacement of dentin by $\mathrm{ZnPO}_{4}$ to a depth of $2 \mathrm{~mm}$ does not result in any significant increase in the tensile stress induced in the amalgam. This result is contrary to the postulation of Vieira and Mondelli ${ }^{9}$ who concluded that a decrease in the fracture strength of amalgam must be anticipated for deep cavities that require bases. In contrast, increasing the depth of a ZOE base markedly increased the stresses in the amalgam restoration. It appears that the $\mathrm{ZOE}$, unlike $\mathrm{ZnPO}_{4}$ cement base, does not behave as a rigid material in dissipating the stress to the surrounding tissue, but transmits it more directly to the cavity floor and induces a larger displacement. In comparison with $\mathrm{ZnPO}_{4}$ cement base, this material does not have adequate mechanical properties to support a restoration and what is required is the develop-

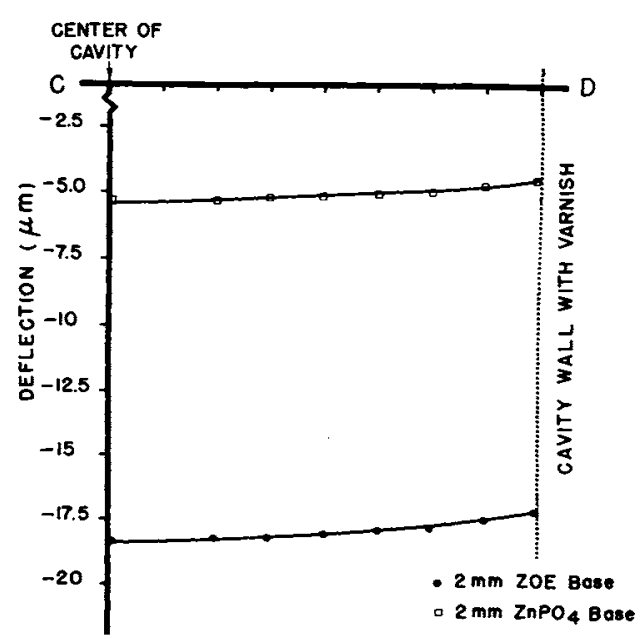

Fig 5.-Deflection along line $C-D$ at amalgambase interface. ment of a cement base with similar mechanical properties to $\mathrm{ZnPO}_{4}$, particularly the modulus of elasticity, but without the deleterious biological properties. Even thin layers of ZOE $(0.5 \mathrm{~mm})$ caused significant changes in the stress induced in the amalgam, and if all other factors are equal, it is better from a mechanical standpoint to place the restoration on a dentin floor rather than interpose a thin layer of ZOE cement base.

The low strength of $\mathrm{ZnO}$ cement and its inability to resist condensation has been recognized, and the use of composite bases has been recommended. Both this study and that of $\mathrm{Luke}^{7}$ indicate that this is preferential to the use of ZOE alone. It appears that the increased support offered by the combined $\mathrm{ZnPO}_{4}$ and $\mathrm{ZOE}$ base over an equivalent thickness of $\mathrm{ZOE}$ is due to a reduction in the thickness of the ZOE.

It has been considered by some authors ${ }^{15,16}$ that the compressive strength of the base was the most important property in its ability to support the restoration, but this has been questioned by Phillips. ${ }^{17} \mathrm{He}$ considered that the base itself was not a contributing factor in the fracture of amalgam restorations and that it was doubtful whether the strength of the base played any role in the fracture. This study indicates that it is the modulus of elasticity of the base material that influences the fracture strength of the amalgam, and hence the base itself was of prime importance. Although a low value for compressive strength is undesirable, indefinitely increasing the compressive strength will not necessarily enhance the ability of the base to support the amalgam restoration. The support provided to the restoration is produced more by the modulus of the base and less by its compressive strength. It follows that fracture of the base does not necessarily precede fracture of the amalgam restoration.

In clinical dentistry the use of a base is frequently necessary to fill a deep central excavation to the level of the cavity floor. Although the experimental model used by Hoppenstand and McConnells did not determine a decrease in strength when ZOE base was used in place of a $\mathrm{ZnPO}_{4}$ base in a central excavation, these differences were readily distinguishable by the present model (Fig 4). As in previous instances no dif- 
ference between a flat dentin floor and a dentin floor with a deep central excavation was evident when this excavation was filled with $\mathrm{ZnPO}_{4}$, but again there was a noticeable difference when ZOE was used in the excavation.

Comparison of the stresses induced in the amalgam restoration when the cavity floor contains a central area of enamel (case 8) and when the cavity floor is of equal depth but composed only of dentin showed that the former induced less stress in the amalgam restoration. It is presumed that the higher modulus of the enamel provides support in the area where the deflection of the amalgam would normally be the greatest. Thus, it reduces the induced tensile stresses in the amalgam. Although it is not recom. mended that enamel be left in a cavity floor for support, the results indicate that if the cavity has sufficient depth for an amalgam restoration and some enamel is still present, it will not contribute to the mechanical failure of the restoration but will reduce the tensile stress at the amalgam tooth interface. The effect of the modulus on the deflection in the amalgam was again observed in Figure 5. A noticeable increase from approximately 5 to $18 \mu \mathrm{m}$ was encountered when the base supporting the amalgam was changed from a $\mathrm{ZnPO}_{4}$ to a $\mathrm{ZOE}$ cement base. The results imply that if all other factors are equal, the probability of amalgam fracturing when supported by a $\mathrm{ZOE}$ base is at least three to four times higher than that of amalgam supported by $\mathrm{ZnPO}_{4}$ base.

This study, together with previous ones, shows that the nature of the base influences the fracture of the overlaying amalgam, but it is probable that clinical failure is modified by other contributing factors such as insufficient depth of the amalgam, stress concentrations caused by the carving of sharp, deep, anatomical contours in the amalgam surface, and premature occlusal contacts. The main advantage of the model used in this study is that the magnitude, direction, and location of the load are repeatable, and the thickness is easily controllable. Thus, the results are reproducible. Since all other experimental variables are controlled, the results depend strictly on the mechanical properties of the bases and reflect the behavior of the materials.

\section{Conclusions}

A computerized dental model was used to study the stress induced in a Class 1 amalgam restoration when supported by constant thickness of $\mathrm{ZOE}$ or $\mathrm{ZnPO}_{4}$ cement bases, or combinations of thicknesses of these bases. The modulus of these bases played an important role in the magnitudes of the stress found in the amalgam restoration. For example, when ZOE was used as a base the maximum tensile stress at the center of the cavity preparation was $35 \mathrm{MN} / \mathrm{m}^{2}$. However, the maximum tensile stress in the amalgam was only $9 \mathrm{MN} / \mathrm{m}^{2}$ when $\mathrm{ZnPO}_{4}$ was used as a base. Similarly, the maximum deflection at the center of the amalgam restoration increased from 5 to $18 \mu \mathrm{m}$ when $\mathrm{ZnPO}_{4}$ and $\mathrm{ZOE}$ cement bases were used, respectively.

\section{References}

1. Cartron, M.L.: Base Materials for Amalgam Restorations, Tex Dent $J$ 79: 4-6, 1961

2. Chong, W.F.; Swartz, M.L.; and Phillips, R.W.: Displacement of Cement Bases by Amalgam Condensation, JADA 74:97-102, 1967.

3. Plant, C.G., and Wrison, H.J.: Forces Exerted on Lining Materials, $B r$ Dent $J$ 131: $62-66,1971$.

4. EL-Bahloul, A.N.: An Evaluation of Insulating and Base Materials, Egypt Dent $J$ 17: 23-32, 1971 .

5. Hoppenstand, D.C., and McConnell, D.: Mechanical Failure of Amalgam Restorations with Zinc Phosphate and Zinc OxideEugenol Cement Bases, J Dent Res 39:899$905,1960$.

6. Rowe, W.C.: How a Base Affects Fracture Resistance of Amalgam, I Dent Child 31: $187.191,1964$.

7. LUKE, L.S.: Impact Loading of Amalgam Supported by Bases of Varying Compressive Strengths, J Dent Child 39: 308-312, 1972.

8. STERN, G.A.: Effects of Impact Loading of Class II Amalgam Restorations in Acrylic Resin Teeth Supported by Various Bases, MSc thesis, School of Dentistry, University of Michigan, 1972.

9. Vieira, D.F., and Mondelli, J: Fracture Strength of Class II Amalgam Restorations Condensed Over Protective Bases, $J$ Prosthet Dent 30: 166-172, 1973.

10. Farah, J.W.; Hood, J.A.; and Craig, R.G.: Stress and Deflections in the Floor of Model Cavity Preparations, $J$ Oral Rehab 1: 207-15, 1974.

11. Farah, J.W.: Stress Analysis of First Molars 
with Full-Crown Preparations by ThreeDimensional Photoelasticity and the Finite Element Method, PhD dissertation, University of Michigan, 1972.

12. Craic, R.G.; Peyton, F.A.; and Johnson, D.W.: Compressive Properties of Enamel, Dental Cements and Gold, I Dent Res 40: 936-945, 1961 .

13. Hall, D.R.; Nakayama, W.T.; Grenoble, D.E.; and KaTz, J.L.: Elastic Constants of Three Representative Dental Cements, $J$ Dent Res 52: 390, 1973.

14. Tylman, S.D.: Theory and Practice of Crown and Fixed Partial Prosthodontics (Bridge), 6th ed, St. Louis: C. V. Mosby Co., 1970, pp 322-324.

15. Mosteller, J.H.: An Evaluation of Intermediate Base Materials, JADA 43: 571-577, 1951.

16. Lyell, J.S.: Base Forming Materials for Restoration of Silver Amalgam, Aust Dent $J$ 5: 132-134, 1960.

17. Phillips, R.W.: Cavity Varnishes and Bases, Dent Clin North Am, March 1965, pp 159168. 